\title{
Stability and Bifurcation of Two Kinds of Three-Dimensional Fractional Lotka-Volterra Systems
}

\author{
Jinglei Tian, Yongguang Yu, and Hu Wang \\ Department of Mathematics, Beijing Jiaotong University, Beijing 100044, China \\ Correspondence should be addressed to Yongguang Yu; ygyu@bjtu.edu.cn
}

Received 23 October 2013; Revised 12 January 2014; Accepted 17 January 2014; Published 12 March 2014

Academic Editor: Yuncai Wang

Copyright (c) 2014 Jinglei Tian et al. This is an open access article distributed under the Creative Commons Attribution License, which permits unrestricted use, distribution, and reproduction in any medium, provided the original work is properly cited.

\begin{abstract}
Two kinds of three-dimensional fractional Lotka-Volterra systems are discussed. For one system, the asymptotic stability of the equilibria is analyzed by providing some sufficient conditions. And bifurcation property is investigated by choosing the fractional order as the bifurcation parameter for the other system. In particular, the critical value of the fractional order is identified at which the Hopf bifurcation may occur. Furthermore, the numerical results are presented to verify the theoretical analysis.
\end{abstract}

\section{Introduction}

In recent years, fractional calculus has attracted much attention of researchers. It has been pointed out that fractional calculus plays an outstanding role in modelling and simulation of systems, such as viscoelastic systems, dielectric polarization, electromagnetic waves, heat conduction, robotics, and biological systems. In fact, fractional derivatives provide an excellent instrument for the description of memory and hereditary properties of various materials and processes in comparison with the classical integer-order counterparts. Therefore, it may be more important and useful to investigate the fractional systems.

Traditionally, the fractional differential equation defined by mathematicians is a Riemann-Liouville fractional derivative [1]. But this definition is less popular because of the fact that it requires initial conditions to be expressed in terms of fractional integrals and their derivatives. Meanwhile, there is no known physical interpretation for such types of initial conditions. In contrast, the alternative definition of the fractional derivative given by Caputo [2] has the advantage of only requiring the initial conditions given in terms of integer-order derivatives. These initial conditions of integerorder derivatives can be measured accurately and represent well-understood features of a physical situation. In [2], it has been pointed out that Caputo's derivative is equivalent to the Riemann-Liouville derivative under homogeneous initial conditions and some smoothness conditions. Therefore, Caputo's definition of fractional derivative is used throughout in this paper.

As is well known, in the field of mathematical biology, the traditional Lotka-Volterra systems are very important mathematical models which describe multispecies population dynamics in a nonautonomous environment. Many important and interesting results on the dynamical behaviors for the Lotka-Volterra systems have been found in [39], such as the existence and uniqueness of solutions, the permanence, extinction, global asymptotic behavior, and bifurcation. Because of the good memory and hereditary properties of fractional derivatives, it is often necessary to study the corresponding fractional systems. Therefore, the dynamical analysis of the fractional Lotka-Volterra systems has attracted a great deal of attention due to its theoretical and practical significance.

Many important results regarding stability of fractional systems have been obtained. For instance, the stability, existence, uniqueness, and numerical solution of the fractional logistic equation are investigated in [10]. The stability and solutions of fractional predator-prey and rabies models are discussed in [11]. In addition, bifurcation properties of fractional systems have been studied in some papers. For example, conditions for the occurrence of Hopf's bifurcation 
are explored based on numerical simulations in [12]. The critical values of the fractional order are identified for which Hopf's bifurcation may occur based on the stability analysis in [13]. Thus, it is significant to study the dynamical behaviors in the fractional population systems.

To the best of our knowledge, some papers have concentrated on the dynamic investigation of the fractional population systems $[10,11]$. However, there are few results on bifurcation phenomena of the fractional population systems. Therefore, in the paper, we mainly consider stability and bifurcation in the three-dimensional fractional LotkaVolterra systems.

Motivated by the above discussions, some dynamical properties of two kinds of three-dimensional fractional Lotka-Volterra systems are investigated in this paper. Existence and uniqueness of solutions are considered. Some sufficient conditions are provided for the asymptotic stability of equilibria. Specifically, bifurcation behaviors are analyzed by formulating the critical values of the fractional order at which Hopf's bifurcations may take place.

The rest of this paper is organized as follows. In Section 2, a three-dimensional fractional Lotka-Volterra predator-prey system with interspecific competition is introduced. And the asymptotic stability of the system is studied. In Section 3, a three-dimensional fractional Lotka-Volterra predator-prey system is provided, and bifurcation properties are investigated. The numerical results in Section 4 are given to verify the theoretical findings. Finally, the paper is concluded in Section 5 .

\section{Stability Analysis of a Three-Dimensional Fractional Lotka-Volterra Predator-Prey System with Interspecific Competition}

Consider a three-dimensional fractional Lotka-Volterra system

$$
\begin{aligned}
& D^{q} x_{1}(t)=x_{1}(t)\left(b_{1}-a_{11} x_{1}(t)-a_{12} x_{2}(t)-a_{13} x_{3}(t)\right), \\
& D^{q} x_{2}(t)=x_{2}(t)\left(-b_{2}+a_{21} x_{1}(t)-a_{22} x_{2}(t)-a_{23} x_{3}(t)\right), \\
& D^{q} x_{3}(t)=x_{3}(t)\left(-b_{3}+a_{31} x_{1}(t)-a_{32} x_{2}(t)-a_{33} x_{3}(t)\right),
\end{aligned}
$$

with the initial values $\left.x_{i}(t)\right|_{t=0}=x_{i}(0), i=1,2,3$, where $0<q \leq 1$; especially when $q=1$, the system (1) is a classical integer-order system. All constant coefficients $a_{i j}$ and $b_{i}(i, j=$ $1,2,3)$ can be arbitrary positive real numbers. $x_{1}(t) \geq 0$ represents the density of prey species at time $t$, and $x_{2}(t) \geq 0$, $x_{3}(t) \geq 0$ represent the densities of predator species at time $t$. In this case, system (1) can be regarded as a fractional LotkaVolterra predator-prey system with interspecific competition.

In the following, existence and uniqueness of solutions for system (1) are given. In addition, the important results related to the stability of the fractional systems are presented to provide the theoretical bases for the further study.
Here, the fractional Lotka-Volterra system (1) can be rewritten in the form

$$
\begin{gathered}
D^{q} X(t)=A X(t)+x_{1}(t) B_{1} X(t) \\
+x_{2}(t) B_{2} X(t)+x_{3}(t) B_{3} X(t), \\
X(0)=X_{0},
\end{gathered}
$$

where $0<q \leq 1, t \in(0, T]$, and

$$
\begin{gathered}
X(t)=\left(\begin{array}{l}
x_{1}(t) \\
x_{2}(t) \\
x_{3}(t)
\end{array}\right), \quad X_{0}=\left(\begin{array}{l}
x_{1}(0) \\
x_{2}(0) \\
x_{3}(0)
\end{array}\right), \\
A=\left(\begin{array}{ccc}
b_{1} & 0 & 0 \\
0 & -b_{2} & 0 \\
0 & 0 & -b_{3}
\end{array}\right), \\
B_{1}=\left(\begin{array}{ccc}
-a_{11} & -a_{12} & -a_{13} \\
0 & 0 & 0 \\
0 & 0 & 0
\end{array}\right), \\
B_{3}=\left(\begin{array}{rrr}
0 & 0 & 0 \\
0 & 0 & 0 \\
a_{31} & -a_{32} & -a_{33}
\end{array}\right) .
\end{gathered}
$$

Definition 1. For $X(t)=\left(x_{1}(t), x_{2}(t), x_{3}(t)\right)^{T}$, let $C^{*}[0, T]$ be the set of continuous column vectors $X(t)$ on the interval $[0, T]$. The norm of $X(t) \in C^{*}[0, T]$ is given by $\|X(t)\|=$ $\sum_{i=1}^{3} \sup _{t}\left|x_{i}(t)\right|$.

Theorem 2. System (2) has a unique solution if $X(t) \in$ $C^{*}[0, T]$.

Proof. Let $F(X(t))=A X(t)+x_{1}(t) B_{1} X(t)+x_{2}(t) B_{2} X(t)+$ $x_{3}(t) B_{3} X(t)$, then $X(t) \in C^{*}[0, T]$ implies $F(X(t)) \in$ $C^{*}[0, T]$. In addition, take $X(t), Y(t) \in C^{*}[0, T]$ and $X(t) \neq Y(t)$; the following inequality holds:

$$
\begin{aligned}
& \|F(X(t))-F(Y(t))\| \\
& =\| A(X(t)-Y(t))+x_{1}(t) B_{1} X(t) \\
& \quad-y_{1}(t) B_{1} Y(t)+x_{2}(t) B_{2} X(t) \\
& \quad-y_{2}(t) B_{2} Y(t)+x_{3}(t) B_{3} X(t) \\
& \quad-y_{3}(t) B_{3} Y(t) \| \\
& =\| A(X(t)-Y(t))+x_{1}(t) B_{1}(X(t)-Y(t)) \\
& \quad+\left(x_{1}(t)-y_{1}(t)\right) B_{1} Y(t)+x_{2}(t) B_{2}(X(t)-Y(t)) \\
& \quad+\left(x_{2}(t)-y_{2}(t)\right) B_{2} Y(t)+x_{3}(t) B_{3}(X(t)-Y(t)) \\
& \quad+\left(x_{3}(t)-y_{3}(t)\right) B_{3} Y(t) \| \\
& \leq\|A(X(t)-Y(t))\|+\left\|x_{1}(t) B_{1}(X(t)-Y(t))\right\| \\
& +\left\|\left(x_{1}(t)-y_{1}(t)\right) B_{1} Y(t)\right\|+\left\|x_{2}(t) B_{2}(X(t)-Y(t))\right\| \\
& +\left\|\left(x_{2}(t)-y_{2}(t)\right) B_{2} Y(t)\right\|+\left\|x_{3}(t) B_{3}(X(t)-Y(t))\right\|
\end{aligned}
$$




$$
\begin{aligned}
& \quad+\left\|\left(x_{3}(t)-y_{3}(t)\right) B_{3} Y(t)\right\| \\
& \leq\left(\|A\|+\left\|B_{1}\right\|\left(\left|x_{1}(t)\right|+\|Y(t)\|\right)\right. \\
& \quad+\left\|B_{2}\right\|\left(\left|x_{2}(t)\right|+\|Y(t)\|\right) \\
& \left.\quad+\left\|B_{3}\right\|\left(\left|x_{3}(t)\right|+\|Y(t)\|\right)\right)\|X(t)-Y(t)\| \\
& \leq L\|X(t)-Y(t)\|,
\end{aligned}
$$

where $L=\|A\|+\left(\left\|B_{1}\right\|+\left\|B_{2}\right\|+\left\|B_{3}\right\|\right)\left(M_{1}+M_{2}\right)>0$, and $M_{1}$ and $M_{2}$ are positive and satisfy $\|X(t)\| \leq M_{1},\|Y(t)\| \leq M_{2}$ as a result of $X(t), Y(t) \in C^{*}[0, T]$. Based on Theorems 2.1 and 2.2 in [14], system (2) has a unique solution.

Theorem 3 (see [15]). The linear autonomous system $D^{q} x=$ Ax is asymptotically stable if and only if

$$
|\arg (\lambda)|>\frac{q \pi}{2}
$$

where $A \in R^{n \times n}, q \in(0,1)$, and $\lambda \in \sigma(A) ; \sigma(A)$ denotes the set of all eigenvalues of the matrix $A$.

Theorem 4. Let $x^{*}$ be an equilibrium of the nonlinear system (1), then the equilibrium $x^{*}$ is locally asymptotically stable if

$$
|\arg (\lambda)|>\frac{q \pi}{2}
$$

where $\lambda \in \sigma\left(J\left(x^{*}\right)\right)$; $\sigma\left(J\left(x^{*}\right)\right)$ denotes the set of all eigenvalues of the Jacobian matrix $J\left(x^{*}\right)$.

Proof. The proof follows from Theorem 3 and [11].

In the following, the stability of system (1) is investigated by giving some appropriate conditions. The asymptotic stability of the equilibria is demonstrated based on Theorem 4 . Through simple calculation, the equilibria of system (1) are obtained and denoted as

$$
\begin{aligned}
& P_{1}=(0,0,0), \quad P_{2}=\left(0,0,-\frac{b_{3}}{a_{33}}\right), \\
& P_{3}=\left(0,-\frac{b_{2}}{a_{22}}, 0\right), \quad P_{4}=\left(\frac{b_{1}}{a_{11}}, 0,0\right), \\
& P_{5}=\left(0, \frac{c_{52}}{c_{5}}, \frac{c_{53}}{c_{5}}\right), \quad P_{6}=\left(\frac{c_{61}}{c_{6}}, 0, \frac{c_{63}}{c_{6}}\right) \text {, } \\
& P_{7}=\left(\frac{c_{71}}{c_{7}}, \frac{c_{72}}{c_{7}}, 0\right), \quad P_{8}=\left(\frac{c_{81}}{c_{8}}, \frac{c_{82}}{c_{8}}, \frac{c_{83}}{c_{8}}\right) \text {, }
\end{aligned}
$$

where $c_{52}=b_{3} a_{23}-b_{2} a_{33}, c_{53}=b_{2} a_{32}-b_{3} a_{22}, c_{5}=a_{22} a_{33}-$ $a_{23} a_{32}, \quad c_{61}=b_{1} a_{33}+b_{3} a_{13}, c_{63}=b_{1} a_{31}-b_{3} a_{11}, c_{6}=$ $a_{11} a_{33}+a_{13} a_{31}, \quad c_{71}=b_{1} a_{22}+b_{2} a_{12}, \quad c_{72}=b_{1} a_{21}-b_{2} a_{11}, c_{7}=$ $a_{11} a_{22}+a_{12} a_{21}, \quad c_{81}=b_{1} a_{22} a_{33}-b_{1} a_{23} a_{32}+b_{2} a_{12} a_{33}-b_{2} a_{13} a_{32}+$ $b_{3} a_{13} a_{22}-b_{3} a_{12} a_{23}, \quad c_{82}=b_{1} a_{21} a_{33}-b_{1} a_{23} a_{31}-b_{2} a_{11} a_{33}-$ $b_{2} a_{13} a_{31}+b_{3} a_{11} a_{23}+b_{3} a_{13} a_{21}, c_{83}=b_{1} a_{22} a_{31}-b_{1} a_{21} a_{32}+$ $b_{2} a_{11} a_{32}+b_{2} a_{12} a_{31}-b_{3} a_{11} a_{22}-b_{3} a_{12} a_{21}$, and $c_{8}=a_{11} a_{22} a_{33}-$ $a_{11} a_{23} a_{32}+a_{12} a_{21} a_{33}-a_{12} a_{23} a_{31}+a_{13} a_{22} a_{31}-a_{13} a_{21} a_{32}$.
Because of the fact that all constant coefficients of system (1) are positive, $P_{2}, P_{3}$, and $P_{5}$ are in contradiction with the actual situation; hence the asymptotical stability of other five equilibria will be studied in detail.

Theorem 5. For the three-dimensional fractional LotkaVolterra system (1), the following results can be obtained.

(a) $P_{1}$ is unstable;

(b) $P_{4}$ is locally asymptotically stable if $b_{1} / a_{11}<b_{2} / a_{21}$, $b_{1} / a_{11}<b_{3} / a_{31}$

(c) $P_{6}$ is locally asymptotically stable if $b_{3} / a_{31}<b_{1} / a_{11}<$ $b_{2} / a_{21}$

(d) $P_{7}$ is locally asymptotically stable if $b_{2} / a_{21}<b_{1} / a_{11}<$ $b_{3} / a_{31}$;

(e) $P_{8}$ is locally asymptotically stable if $a_{32} / a_{22}<a_{31} / a_{21}<$ $a_{33} / a_{23}$.

Proof. For $P_{1}=(0,0,0)$, its Jacobian matrix is

$$
J\left(P_{1}\right)=\left(\begin{array}{ccc}
b_{1} & 0 & 0 \\
0 & -b_{2} & 0 \\
0 & 0 & -b_{3}
\end{array}\right),
$$

and the eigenvalues of $J\left(P_{1}\right)$ satisfy $\lambda_{1}=b_{1}>0, \lambda_{2}=-b_{2}<$ 0 , and $\lambda_{3}=-b_{3}<0$; hence the equilibrium $P_{1}$ is unstable.

For $P_{4}$, its Jacobian matrix is

$$
J\left(P_{4}\right)=\left(\begin{array}{ccc}
-b_{1} & -\frac{b_{1} a_{12}}{a_{11}} & -\frac{b_{1} a_{13}}{a_{11}} \\
0 & \frac{b_{1} a_{21}-b_{2} a_{11}}{a_{11}} & 0 \\
0 & 0 & \frac{b_{1} a_{31}-b_{3} a_{11}}{a_{11}}
\end{array}\right),
$$

and the eigenvalues of $J\left(P_{4}\right)$ satisfy $\lambda_{1}=-b_{1}<0, \lambda_{2}=$ $\left(b_{1} a_{21}-b_{2} a_{11}\right) / a_{11}<0$, and $\lambda_{3}=\left(b_{1} a_{31}-b_{3} a_{11}\right) / a_{11}<0$; hence the equilibrium $P_{4}$ is locally asymptotically stable.

For $P_{6}$, use the notations below:

$$
J\left(P_{6}\right)=\left(\begin{array}{ccc}
A_{11} & A_{12} & A_{13} \\
0 & A_{22} & 0 \\
A_{31} & A_{32} & A_{33}
\end{array}\right),
$$

and its characteristic equation is

$$
\left(\lambda-A_{22}\right)\left(\lambda^{2}-\left(A_{11}+A_{33}\right) \lambda+A_{11} A_{33}-A_{13} A_{31}\right)=0 .
$$

Based on the condition from (c), the following formulas can be easily got

$$
\begin{gathered}
A_{11}=\frac{c_{11}}{c_{0}}<0, \quad A_{13}=\frac{c_{13}}{c_{0}}<0, \\
A_{31}=\frac{c_{31}}{c_{0}}>0, \quad A_{22}=\frac{c_{22}}{c_{0}}<0, \\
A_{33}=\frac{c_{33}}{c_{0}}<0,
\end{gathered}
$$


where $c_{11}=-b_{1} a_{11} a_{33}-b_{3} a_{11} a_{13}, c_{13}=-b_{1} a_{13} a_{33}-$ $b_{3} a_{13} a_{13}, c_{31}=b_{1} a_{31} a_{31}-b_{3} a_{11} a_{31}, c_{22}=a_{33}\left(b_{1} a_{21}-b_{2} a_{11}\right)+$ $a_{23}\left(b_{3} a_{11}-b_{1} a_{31}\right)+a_{13}\left(b_{3} a_{21}-b_{2} a_{31}\right), c_{33}=-b_{1} a_{31} a_{33}+b_{3} a_{11} a_{33}$, and $c_{0}=a_{11} a_{33}+a_{13} a_{31}$. Then, the following results can be obtained:

$$
\begin{gathered}
\lambda_{1}=A_{22}<0, \quad \lambda_{2}+\lambda_{3}=A_{11}+A_{33}<0, \\
\lambda_{2} \lambda_{3}=A_{11} A_{33}-A_{13} A_{31}>0 .
\end{gathered}
$$

Hence the equilibrium $P_{6}$ is locally asymptotically stable.

Similarly, it can be readily derived that the equilibrium $P_{7}$ is locally asymptotically stable.

For $P_{8}$, let $P_{8}=\left(x_{1}^{*}, x_{2}^{*}, x_{3}^{*}\right)$; the Jacobian matrix of $P_{8}$ can be written as

$$
J\left(P_{8}\right)=\left(\begin{array}{ccc}
-a_{11} x_{1}^{*} & -a_{12} x_{1}^{*} & -a_{13} x_{1}^{*} \\
a_{21} x_{2}^{*} & -a_{22} x_{2}^{*} & -a_{23} x_{2}^{*} \\
a_{31} x_{3}^{*} & -a_{32} x_{3}^{*} & -a_{33} x_{3}^{*}
\end{array}\right)=\left(\begin{array}{lll}
B_{11} & B_{12} & B_{13} \\
B_{21} & B_{22} & B_{23} \\
B_{31} & B_{32} & B_{33}
\end{array}\right),
$$

and its characteristic equation is

$$
\lambda^{3}+C_{1} \lambda^{2}+C_{2} \lambda+C_{3}=0
$$

where $C_{1}=-\left(B_{11}+B_{22}+B_{33}\right), C_{2}=B_{11} B_{22}+B_{11} B_{33}+$ $B_{22} B_{33}-B_{23} B_{32}-B_{12} B_{21}-B_{13} B_{31}$, and $C_{3}=-B_{11} B_{22} B_{33}+$ $B_{11} B_{23} B_{32}+B_{12} B_{21} B_{33}+B_{13} B_{22} B_{31}-B_{12} B_{23} B_{31}-B_{13} B_{21} B_{32}$. For simplicity, the equivalent characteristic equation is introduced as follows:

$$
\lambda^{3}+a_{1} \lambda^{2}+a_{2} \lambda+a_{3}=(\lambda-a)\left(\lambda^{2}-b \lambda+c\right)=0 .
$$

On the basis of the above equivalent substitutions, the following inequalities can be gained:

$$
\begin{gathered}
a_{1}=a_{11} x_{1}^{*}+a_{22} x_{2}^{*}+a_{33} x_{3}^{*}>0 \\
a_{2}=\left(a_{11} a_{22}+a_{12} a_{21}\right) x_{1}^{*} x_{2}^{*}+\left(a_{11} a_{33}+a_{13} a_{31}\right) x_{1}^{*} x_{3}^{*} \\
+\left(a_{22} a_{33}-a_{23} a_{32}\right) x_{2}^{*} x_{3}^{*}>0 \\
a_{3}=\left(a_{11}\left(a_{22} a_{33}-a_{23} a_{32}\right)+a_{12}\left(a_{21} a_{33}-a_{23} a_{31}\right)\right. \\
\left.+a_{13}\left(a_{22} a_{31}-a_{21} a_{32}\right)\right) x_{1}^{*} x_{2}^{*} x_{3}^{*}>0, \\
a_{1} a_{2}-a_{3}=d_{1} x_{1}^{*} x_{2}^{*} x_{3}^{*}+d_{2} x_{1}^{*} x_{2}^{* 2}+d_{3} x_{1}^{*} x_{3}^{* 2} \\
+d_{4} x_{1}^{* 2} x_{2}^{*}+d_{5} x_{1}^{* 2} x_{3}^{*} \\
+d_{6}\left(a_{22} x_{2}^{* 2} x_{3}^{*}+a_{33} x_{2}^{*} x_{3}^{* 2}\right)>0 \\
a+b=-a_{1}<0 \\
a b+c=a_{2}>0 \\
a c=-a_{3}<0 \\
-a^{2} b-a b^{2}-b c=a_{1} a_{2}-a_{3}>0,
\end{gathered}
$$

where $d_{1}=2 a_{11} a_{22} a_{33}+a_{12} a_{23} a_{31}+a_{13} a_{21} a_{32}, d_{2}=$ $a_{11} a_{22}^{2}+a_{12} a_{21} a_{22}, d_{3}=a_{11} a_{33}^{2}+a_{13} a_{31} a_{33}, d_{4}=a_{11}^{2} a_{22}+$ $a_{11} a_{12} a_{21}, d_{5}=a_{11}^{2} a_{33}+a_{11} a_{13} a_{31}$, and $d_{6}=a_{22} a_{33}-a_{23} a_{32}$. Using the proof by contradiction, it can be concluded that the eigenvalues of $J\left(P_{8}\right)$ satisfy

$$
\begin{gathered}
\lambda_{1}=a<0, \\
\lambda_{2}+\lambda_{3}=b<0, \\
\lambda_{2} \lambda_{3}=c>0, \\
\left|\arg \left(\lambda_{i}\right)\right|>\frac{q \pi}{2}, \quad i=2,3 .
\end{gathered}
$$

Hence the equilibrium $P_{8}$ is locally asymptotically stable.

For the further dynamic investigation of the fractional population systems, the other fractional Lotka-Volterra systems will be considered in the following section. Particularly, bifurcation properties for the system will be studied in detail.

\section{Bifurcation Analysis of a Three-Dimensional Fractional Lotka-Volterra Predator-Prey System}

Consider a three-dimensional fractional Lotka-Volterra system:

$$
\begin{gathered}
D^{q} x_{1}(t)=x_{1}(t)\left(b_{1}-a_{11} x_{1}(t)-a_{12} x_{2}(t)-a_{13} x_{3}(t)\right), \\
D^{q} x_{2}(t)=x_{2}(t)\left(-b_{2}+a_{21} x_{1}(t)-a_{22} x_{2}(t)\right), \\
D^{q} x_{3}(t)=x_{3}(t)\left(-b_{3}+a_{31} x_{1}(t)-a_{33} x_{3}(t)\right),
\end{gathered}
$$

with the initial values $\left.x_{i}(t)\right|_{t=0}=x_{i}(0), i=1,2,3$, where $0<q<1, a_{11}<0$, and the other constant coefficients are positive. $x_{1}(t) \geq 0$ represents the density of prey species at time $t$, and $x_{2}(t) \geq 0, x_{3}(t) \geq 0$ represent the densities of predator species at time $t$. In this case, system (19) can be regarded as a fractional Lotka-Volterra predator-prey system.

On the basis of Theorem 2, it is not difficult to prove that system (19) has a unique solution in a similar way.

It is clear that there are eight equilibria for system (19). Here, we focus on the bifurcation investigation of the equilibrium $x^{*}$ which can be called a positive equilibrium when some conditions are satisfied. The equilibrium $x^{*}$ is obtained as

$$
x^{*}=\left(x_{1}^{*}, x_{2}^{*}, x_{3}^{*}\right)=\left(\frac{d_{11}}{d}, \frac{d_{22}}{d}, \frac{d_{33}}{d}\right),
$$

where $d_{11}=b_{1} a_{22} a_{33}+b_{2} a_{12} a_{33}+b_{3} a_{13} a_{22}, d_{22}=b_{1} a_{21} a_{33}-$ $b_{2}\left(a_{11} a_{33}+a_{13} a_{31}\right)+b_{3} a_{13} a_{21}, d_{33}=b_{1} a_{22} a_{31}+b_{2} a_{12} a_{31}-$ $b_{3}\left(a_{11} a_{22}+a_{12} a_{21}\right)$, and $d=a_{11} a_{22} a_{33}+a_{12} a_{21} a_{33}+a_{13} a_{22} a_{31}$. And its Jacobian matrix can be expressed as

$$
J\left(x^{*}\right)=\left(\begin{array}{ccc}
-a_{11} x_{1}^{*} & -a_{12} x_{1}^{*} & -a_{13} x_{1}^{*} \\
a_{21} x_{2}^{*} & -a_{22} x_{2}^{*} & 0 \\
a_{31} x_{3}^{*} & 0 & -a_{33} x_{3}^{*}
\end{array}\right) \text {. }
$$


Furthermore, the eigenvalues of $J\left(x^{*}\right)$ satisfy the characteristic equation

$$
(\lambda-\beta)\left(\lambda^{2}-(\alpha+\beta) \lambda+\alpha \beta-\gamma\right)=0,
$$

where $\alpha=-a_{11} x_{1}^{*}, \beta=-a_{22} x_{2}^{*}=-a_{33} x_{3}^{*}$, and $\gamma=$ $-a_{12} a_{21} x_{1}^{*} x_{2}^{*}-a_{13} a_{31} x_{1}^{*} x_{3}^{*}$.

In the following, by choosing the fractional order $q$ as the bifurcation parameter and analyzing the associated characteristic equation (22) of system (19) at the positive equilibrium, we investigate the bifurcation phenomena of the positive equilibrium of system (19) and obtain the conditions under which system (19) undergoes a Hopf bifurcation.

Proposition 6. The positive equilibrium $x^{*}$ of system (19) is locally asymptotically stable if and only if all the following conditions are satisfied:

(i) $\beta<0$,

(ii) $\alpha \beta-\gamma>0$, and

(iii) $\alpha+\beta<2 \cos (q \pi / 2) \sqrt{\alpha \beta-\gamma}$.

Proof. For the characteristic equation (22), the root $\lambda_{1}=\beta<$ 0 , and $\lambda_{2}, \lambda_{3}$ satisfy the equation $\lambda^{2}-(\alpha+\beta) \lambda+\alpha \beta-\gamma=0$. It is clear that $\left|\arg \left(\lambda_{2,3}\right)\right|>q \pi / 2$ if and only if the conditions (ii) and (iii) hold. Based on Theorem 4, Proposition 6 proves to be true.

In addition, by analyzing the condition (iii) of Proposition 6 in detail, the following results can be gained.

Proposition 7. With respect to system (19), if $\beta<0$ and $\alpha \beta-$ $\gamma>0$, the following statements can be obtained.

(a) If $\alpha+\beta \leq 0$, the equilibrium $x^{*}$ is locally asymptotically stable, for any $q \in(0,1)$.

(b) If $0<\alpha+\beta<2 \sqrt{\alpha \beta-\gamma}$, the equilibrium $x^{*}$ is locally asymptotically stable if and only if $q \in\left(0, q^{*}\right)$, where $q^{*}=(2 / \pi) \arccos ((\alpha+\beta) / 2 \sqrt{\alpha \beta-\gamma})$.

(c) If $\alpha+\beta \geq 2 \sqrt{\alpha \beta-\gamma}$, the equilibrium $x^{*}$ is unstable for any $q \in(0,1)$.

Proof. The conclusions (a) and (c) are obvious. For the statement (b), due to $0<\alpha+\beta<2 \sqrt{\alpha \beta-\gamma}$, the equation $\lambda^{2}-(\alpha+\beta) \lambda+\alpha \beta-\gamma=0$ has two complex roots $\lambda_{2}, \lambda_{3}$, and their real part is $(\alpha+\beta) / 2>0$. Then $\left|\arg \left(\lambda_{i}\right)\right|=$ $\arccos ((\alpha+\beta) / 2 \sqrt{\alpha \beta-\gamma}), i=2$, 3. Besides, according to the condition $\arccos ((\alpha+\beta) / 2 \sqrt{\alpha \beta-\gamma})=q^{*} \pi / 2, q \in\left(0, q^{*}\right)$ if and only if $\left|\arg \left(\lambda_{i}\right)\right|>q \pi / 2, i=2,3$. Based on Theorem 4 , it is concluded that Proposition 7 is true.

Remark 8. It is apparent that the critical value satisfies $q^{*} \in$ $(0,1)$. When $q \in\left(0, q^{*}\right), x^{*}$ is locally asymptotically stable; when $q \in\left(q^{*}, 1\right)$, and specially $q=1, x^{*}$ is unstable. That is to say, it has verified that fractional differential equations are, at least, as stable as their integer-order counterparts [4].

Remark 9. Under the situation of statement (b), a bifurcation phenomenon must happen at the critical value $q^{*}$. However, it is difficult to confirm precise bifurcation type. As an interesting bifurcation behavior, Hopf's bifurcation is expected to take place.

According to Proposition 7, if some appropriate conditions about the constant coefficients of system (19) can be found so that statement (b) is satisfied, system (19) will undergo a bifurcation phenomenon. And the critical value $q^{*}$ of the bifurcation parameter $q$ can be expressed by the constant coefficients of system (19). From this, the following theorem is specifically proposed.

Theorem 10. With respect to system (19), if the following conditions are satisfied:

(i) $b_{2}=b_{3}, a_{22}=a_{33}, a_{21}=a_{31}=-a_{11}$;

(ii) $a_{12}+a_{13}-a_{22}>0$,

then the positive equilibrium $x^{*}$ is locally asymptotically stable if and only if $q \in\left(0, q^{*}\right)$, where

$q^{*}$

$$
=\frac{2}{\pi} \arccos \left(b_{2}\left(2 \sqrt{\frac{\left(b_{1}+b_{2}\right)\left(b_{1} a_{22}+b_{2} a_{12}+b_{2} a_{13}\right)}{a_{12}+a_{13}-a_{22}}}\right)^{-1}\right) .
$$

Proof. According to the condition (i), the equilibrium $x^{*}$ can be expressed as

$$
\begin{gathered}
x^{*}=\left(\frac{b_{1} a_{22}+b_{2} a_{12}+b_{2} a_{13}}{a_{11}\left(a_{22}-a_{12}-a_{13}\right)}, \frac{b_{1}+b_{2}}{a_{12}+a_{13}-a_{22}},\right. \\
\left.\frac{b_{1}+b_{2}}{a_{12}+a_{13}-a_{22}}\right) .
\end{gathered}
$$

For (22), the following results can be obtained

$$
\begin{gathered}
\beta=-a_{22} x_{2}^{*}<0, \quad \alpha+\beta=b_{2}>0, \\
\alpha \beta-\gamma=\frac{e_{1}}{a_{12}+a_{13}-a_{22}}>0, \\
4(\alpha \beta-\gamma)-(\alpha+\beta)^{2}=\frac{4 e_{1}}{a_{12}+a_{13}-a_{22}}-b_{2}^{2} \\
=\frac{e_{2}}{a_{12}+a_{13}-a_{22}}>0,
\end{gathered}
$$

where $e_{1}=\left(b_{1}+b_{2}\right)\left(b_{1} a_{22}+b_{2} a_{12}+b_{2} a_{13}\right)$ and $e_{2}=a_{22}\left(4 b_{1}^{2}+\right.$ $\left.b_{2}^{2}\right)+3 b_{2}^{2}\left(a_{12}+a_{13}\right)+4 b_{1} b_{2}\left(a_{12}+a_{13}+a_{22}\right)$. Obviously, the above conclusions satisfy statement (b) of Proposition 7, then it can be derived that

$q^{*}$

$$
=\frac{2}{\pi} \arccos \left(b_{2}\left(2 \sqrt{\frac{\left(b_{1}+b_{2}\right)\left(b_{1} a_{22}+b_{2} a_{12}+b_{2} a_{13}\right)}{a_{12}+a_{13}-a_{22}}}\right)^{-1}\right) .
$$




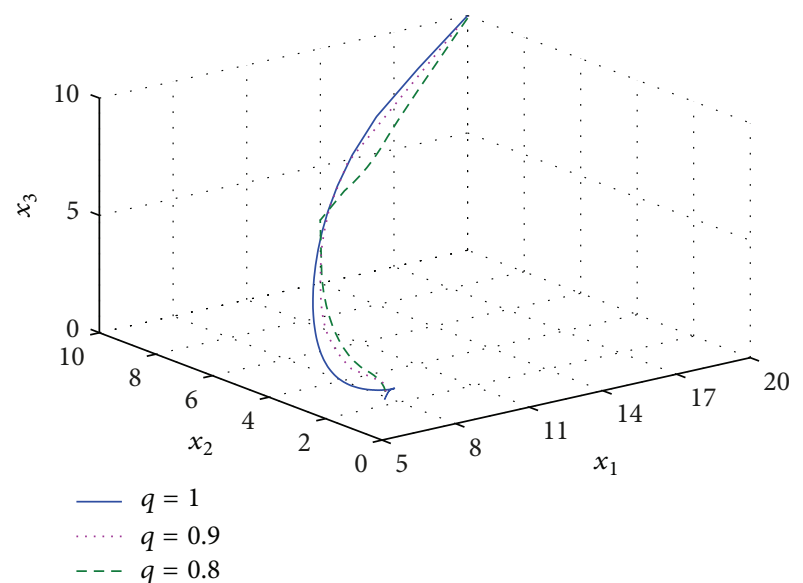

FIgURE 1: The trajectory of system (1) converges to the equilibrium $P_{8}=(53 / 7,15 / 7,1 / 7)$.

Hence, the positive equilibrium $x^{*}$ of system (19) is locally asymptotically stable if and only if $q \in\left(0, q^{*}\right)$.

According to the statement of Theorem 10, it can be concluded that the positive equilibrium $x^{*}$ is locally asymptotically stable if and only if $q \in\left(0, q^{*}\right)$. At $q=q^{*}$, the Hopf bifurcation is expected to take place. As $q$ increases above the critical value $q^{*}$, the positive equilibrium $x^{*}$ is unstable and a limit cycle is expected to appear in the proximity of $x^{*}$ due to the Hopf bifurcation phenomenon.

The analysis of periodic solutions in fractional dynamical systems is a very recent and promising research topic. As a consequence, the nonexistence of exact periodic solutions in time invariant fractional systems is obtained [16]. As an application, it is emphasized that the limit cycle observed in numerical simulations of a simple fractional neural network cannot be an exact periodic solution of the system [17]. In addition, there are some other papers providing the numerical evidences of limit cycles.

Remark 11. Even though exact periodic solutions do not exist in autonomous fractional systems [16, 17], limit cycles have been observed by numerical simulations in many systems such as a fractional neural system [13], a fractional Van der Pol system [18], fractional Chua and Chen's systems [19, 20], and a fractional financial system [21].

\section{Numerical Simulation}

In this paper, an Adams-type predictor-corrector method is used for the numerical solutions of fractional differential equations. This method has been introduced in $[22,23]$ and further investigated in [24-27]. In order to verify the theoretical analysis, the following numerical results are given.

For system (1), the approximate solutions are displayed in Figure 1 for the step size 0.005 and different values of $q, q=1, q=0.9, q=0.8$, respectively. Taking $b_{1}=$ $12, b_{2}=a_{11}=a_{13}=a_{21}=a_{23}=a_{31}=1, a_{12}=$ $a_{32}=a_{33}=2$, and $b_{3}=a_{22}=3$, and choosing the initial values $x_{1}(0)=20, \quad x_{2}(0)=10$, and $x_{3}(0)=10$, the

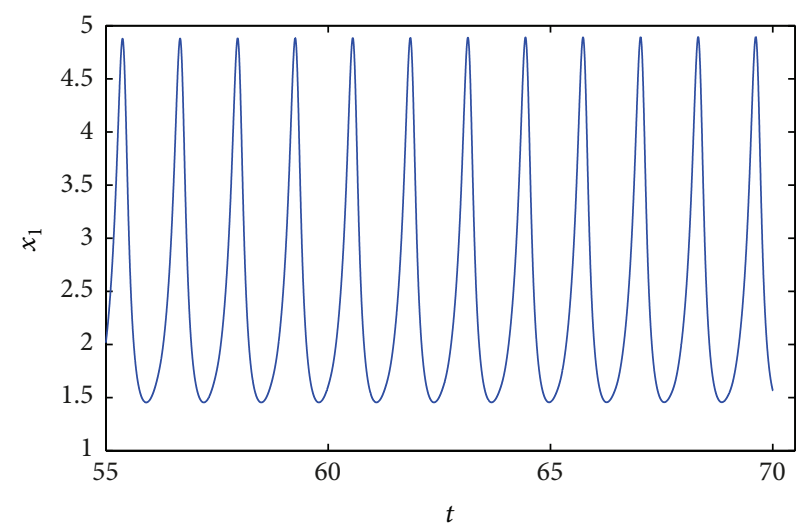

(a)

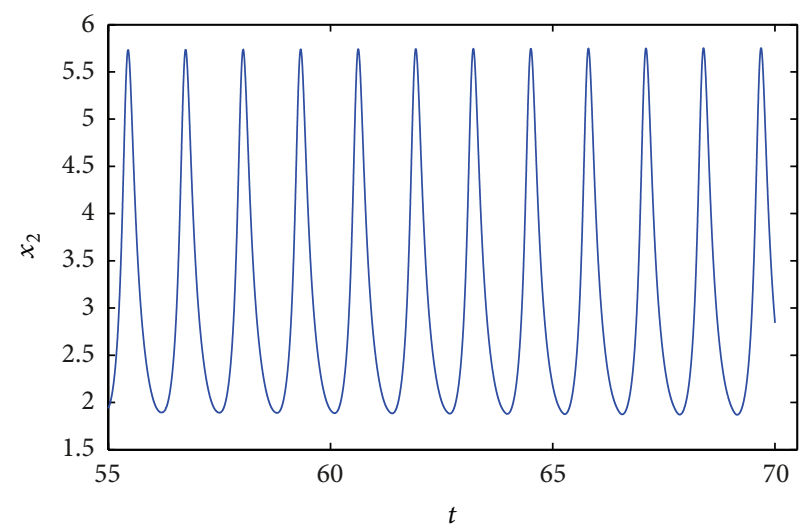

(b)

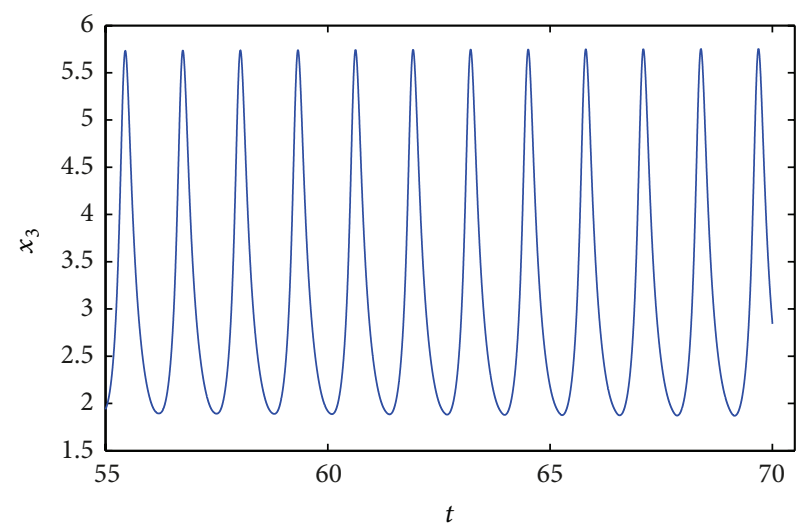

(c)

FIGURE 2: The solution of system (19) versus time with $q=0.84$.

equilibrium $P_{8}$ is $(53 / 7,15 / 7,1 / 7)$. Then, Figure 1 shows that the equilibrium $P_{8}$ is locally asymptotically stable. Namely, the fifth conclusion of Theorem 5 is verified. Similarly, the other conclusions of Theorem 5 can be confirmed.

For system (19), the approximate solutions are displayed in Figures 2, 3, and 4 for the step size 0.001 and different values of $q, q=0.82$, and $q=0.84$. Taking $b_{1}=a_{12}=a_{13}=a_{22}=$ $a_{33}=1, b_{2}=b_{3}=a_{21}=a_{31}=2$, and $a_{11}=-2$, and choosing the initial values $x_{1}(0)=3, x_{2}(0)=x_{3}(0)=4$, the positive equilibrium is $x^{*}=(2.5,3,3)$, and the critical value 


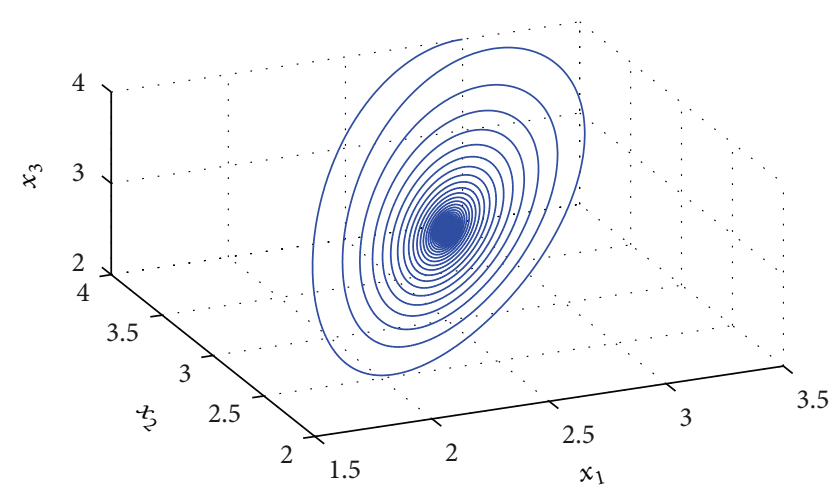

Figure 3: When $q=0.82$, the trajectory of system (19) converges to the equilibrium $x^{*}=(2.5,3,3)$.

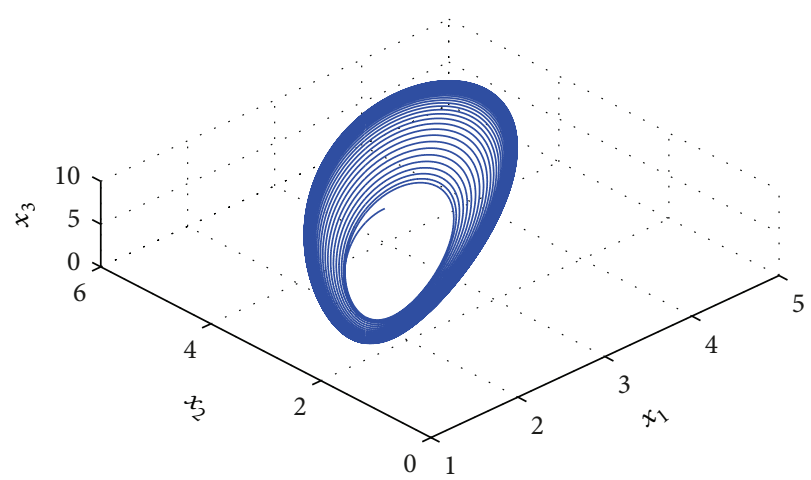

FIGURE 4: When $q=0.84$, the trajectory of system (19) converges to an asymptotically stable limit cycle.

is $q^{*}=0.8337$. Indeed, Figures $2-4$ present the fact that the positive equilibrium $x^{*}$ is locally asymptotically stable when $q=0.82 \in(0,0.8337)$, and when $q=0.84$ increases across $q^{*}=0.8337$, an asymptotically stable limit cycle appears in a neighborhood of the positive equilibrium $x^{*}$.

\section{Conclusion}

In this paper, two kinds of three-dimensional fractional Lotka-Volterra systems have been studied. The main results are divided into two parts. On the one hand, for system (1), the asymptotic stability of the equilibria is investigated by providing simple and reasonable sufficient conditions. And simulation results prove to be quite consistent with the theoretical findings. On the other hand, for system (19), the conditions which could lead to bifurcation phenomena are obtained. Specifically, the fractional order $q \in(0,1)$ is chosen as the bifurcation parameter and the expression of the critical value $q^{*}$ is precisely derived. Furthermore, the numerical result is presented to illustrate that Hopf's bifurcation can take place.

\section{Conflict of Interests}

The authors declare that there is no conflict of interests regarding the publication of this paper.

\section{Acknowledgment}

This work is supported by the National Nature Science Foundation of China under Grant no. 11371049 and the Science Foundation of Beijing Jiaotong University under Grant 2011JBM130.

\section{References}

[1] K. S. Miller and B. Ross, An Introduction to the Fractional Calculus and Fractional Differential Equations, Wiley-Interscience Publication, New York, NY, USA, 1993.

[2] I. Podlubny, Fractional Differential Equations, Academic Press, New York, NY, USA, 1999.

[3] S. Ahmad and A. C. Lazer, "Average conditions for global asymptotic stability in a nonautonomous Lotka-Volterra system," Nonlinear Analysis, Theory, Methods and Applications, vol. 40, no. 1, pp. 37-49, 2000.

[4] S. Ahmad and A. C. Lazer, "Average growth and total permanence in a competitive Lotka-Volterra System," Annali di Matematica Pura ed Applicata, vol. 185, supplement 5, pp. S47S67, 2006.

[5] P. van den Driessche and M. L. Zeeman, "Three-dimensional competitive Lotka-Volterra systems with no periodic orbits," SIAM Journal on Applied Mathematics, vol. 58, no. 1, pp. 227234, 1998.

[6] Z. Teng and L. Chen, "Global asymptotic stability of periodic Lotka-Volterra systems with delays," Nonlinear Analysis: Theory, Methods and Applications, vol. 45, no. 8, pp. 1081-1095, 2001.

[7] N. Fang and X. X. Chen, "Permanence of a discrete multispecies Lotka-Volterra competition predator-prey system with delays," Nonlinear Analysis: Real World Applications, vol. 9, no. 5, pp. 2185-2195, 2008.

[8] G. Lu and Z. Lu, "Permanence for two-species Lotka-Volterra cooperative systems with delays," Mathematical Biosciences and Engineering, vol. 5, no. 3, pp. 477-484, 2008.

[9] X. P. Yan and W. T. Li, "Stability and Hopf bifurcation for a delayed cooperative system with diffusion effects," International Journal of Bifurcation and Chaos, vol. 18, no. 2, pp. 441-453, 2008.

[10] A. M. A. El-Sayed, A. E. M. El-Mesiry, and H. A. A. El-Saka, "On the fractional-order logistic equation," Applied Mathematics Letters, vol. 20, no. 7, pp. 817-823, 2007.

[11] E. Ahmed, A. M. A. El-Sayed, and H. A. A. El-Saka, "Equilibrium points, stability and numerical solutions of fractionalorder predator-prey and rabies models," Journal of Mathematical Analysis and Applications, vol. 325, no. 1, pp. 542-553, 2007.

[12] H. A. El-Saka, E. Ahmed, M. I. Shehata, and A. M. A. El-Sayed, "On stability, persistence, and Hopf bifurcation in fractional order dynamical systems," Nonlinear Dynamics, vol. 56, no. 12, pp. 121-126, 2009.

[13] E. Kaslik and S. Sivasundaram, "Nonlinear dynamics and chaos in fractional-order neural networks," Neural Networks, vol. 32, pp. 245-256, 2012.

[14] K. Diethelm and N. J. Ford, "Analysis of fractional differential equations," Journal of Mathematical Analysis and Applications, vol. 265, no. 2, pp. 229-248, 2002.

[15] D. Matignon, "Stability results for fractional differential equations with applications to control processing," in Proceedings of the Computational Engineering in Systems Applications, vol. 2, pp. 963-968, Lille, France, July 2012. 
[16] M. S. Tavazoei and M. Haeri, "A proof for non existence of periodic solutions in time invariant fractional order systems," Automatica, vol. 45, no. 8, pp. 1886-1890, 2009.

[17] E. Kaslik and S. Sivasundaram, "Non-existence of periodic solutions in fractional-order dynamical systems and a remarkable difference between integer and fractional-order derivatives of periodic functions," Nonlinear Analysis: Real World Applications, vol. 13, no. 3, pp. 1489-1497, 2012.

[18] R. S. Barbosa, J. A. T. MacHado, B. M. Vinagre, and A. J. Calderón, "Analysis of the van der Pol oscillator containing derivatives of fractional order," Journal of Vibration and Control, vol. 13, no. 9-10, pp. 1291-1301, 2007.

[19] D. Cafagna and G. Grassi, "Bifurcation and chaos in the fractional-order Chen system via a time-domain approach," International Journal of Bifurcation and Chaos, vol. 18, no. 7, pp. 1845-1863, 2008.

[20] D. Cafagna and G. Grassi, "Fractional-order Chua's circuit: time-domain analysis, bifurcation, chaotic behavior and test for chaos," International Journal of Bifurcation and Chaos, vol. 18, no. 3, pp. 615-639, 2008.

[21] M. S. Abd-Elouahab, N. E. Hamri, and J. Wang, "Chaos control of a fractional-order financial system," Mathematical Problems in Engineering, vol. 2010, Article ID 270646, 18 pages, 2010.

[22] K. Diethelm and A. Freed, "On the solution of nonlinear fractional order differential equations used in the modelling of viscoplasticity," in Scientific Computing in Chemical Engineering II-Computational Fluid Dynamics, Reaction Engineering, and Molecular Properties, F. Keil, W. Mackens, H. Voß, and J. Werther, Eds., pp. 217-224, Springer, Heidelberg, Germany, 1999.

[23] K. Diethelm and A. Freed, "The FracPECE subroutine for the numerical solution of differential equations of fractional order," in Forschung und Wissenschaftliches Rechnen 1998, S. Heinzel and T. Plesser, Eds., pp. 57-71, Gesellschaft fr Wisseschaftliche Datenverarbeitung, Göttingen, Germany, 1999.

[24] R. Zhao, Dynamical Analysis of Fractional-Order Species Models, College of Science Beijing Jiaotong University, Beijing, China, 2011.

[25] E. Ahmed, A. M. A. El-Sayed, A. E. M. El-Mesiry, and H. A. A. El-Saka, "Numerical solution for the fractional replicator equation," International Journal of Modern Physics C, vol. 16, no. 7, pp. 1017-1025, 2005.

[26] E. Ahmed, A. M. A. El-Sayed, and H. A. A. El-Saka, "On some Routh-Hurwitz conditions for fractional order differential equations and their applications in Lorenz, Rössler, Chua and Chen systems," Physics Letters A, vol. 358, no. 1, pp. 1-4, 2006.

[27] A. E. M. El-Mesiry, A. M. A. El-Sayed, and H. A. A. ElSaka, "Numerical methods for multi-term fractional (arbitrary) orders differential equations," Applied Mathematics and Computation, vol. 160, no. 3, pp. 683-699, 2005. 


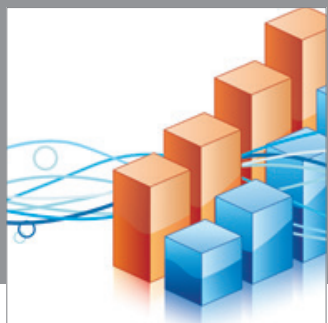

Advances in

Operations Research

mansans

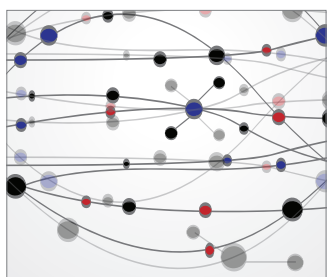

The Scientific World Journal
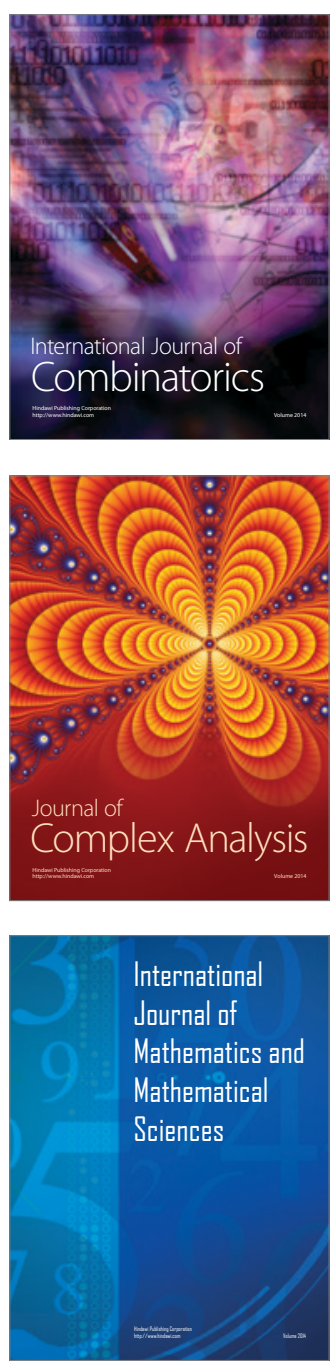
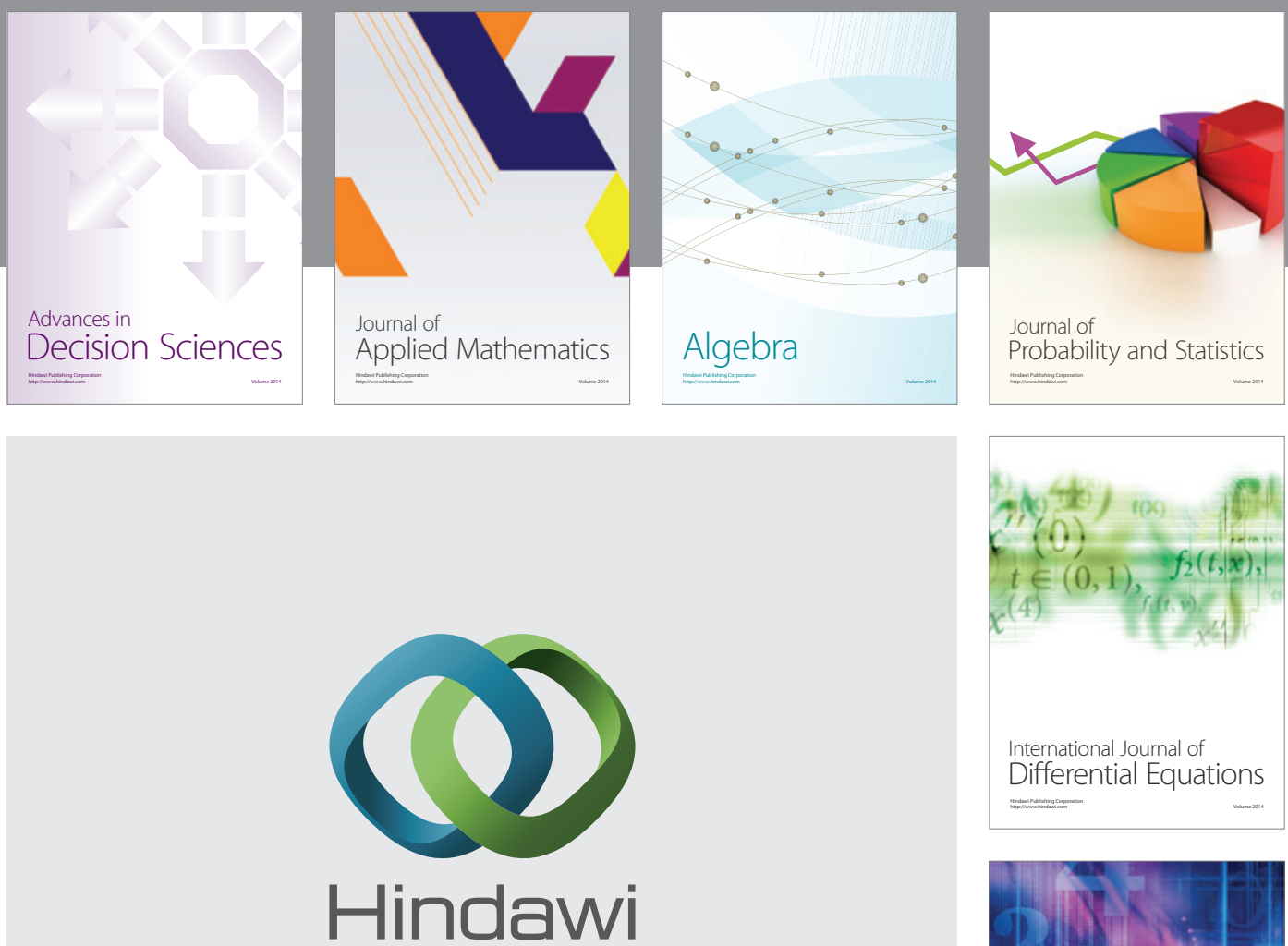

Submit your manuscripts at http://www.hindawi.com
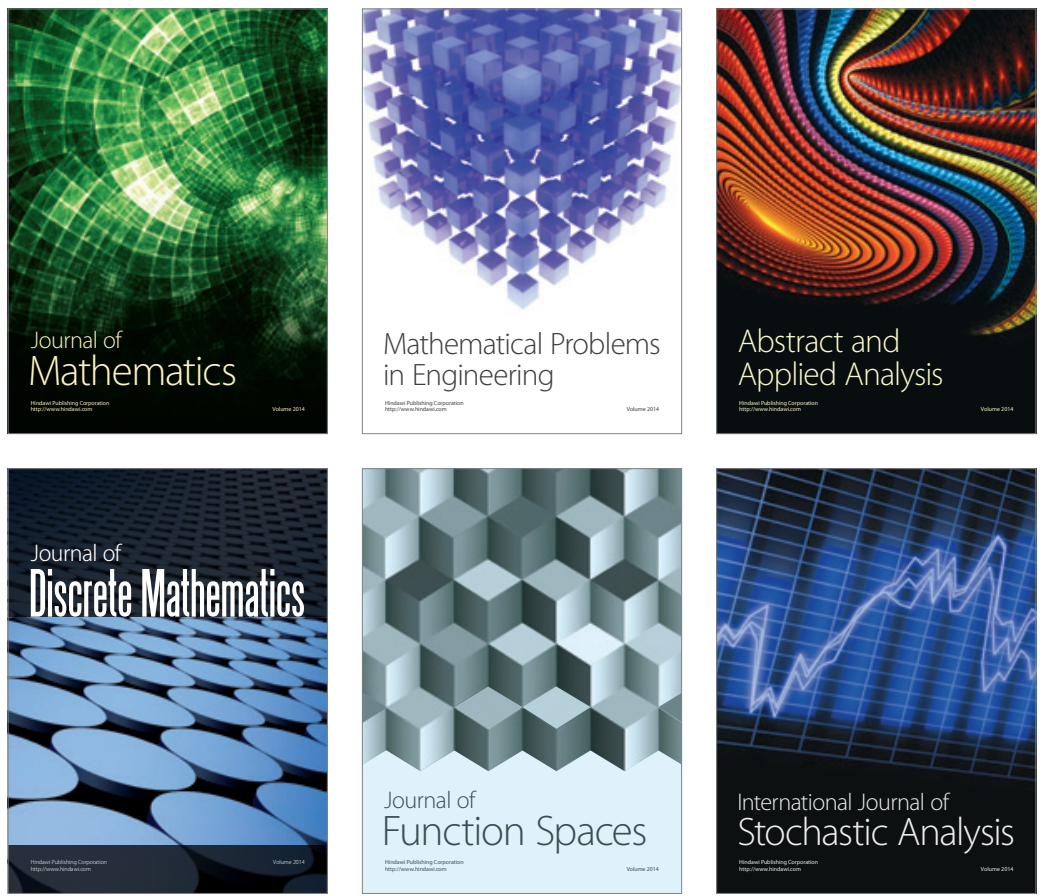

Journal of

Function Spaces

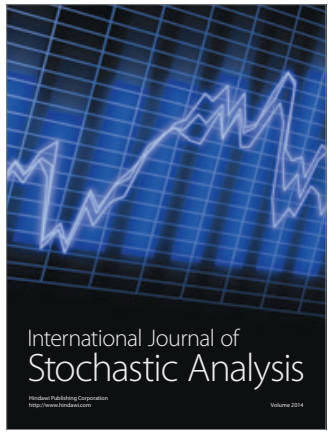

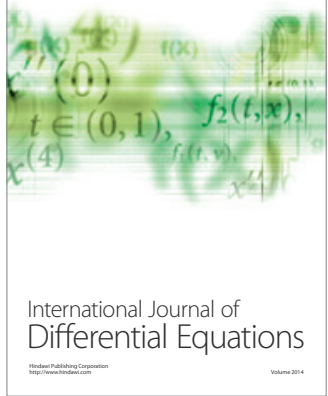
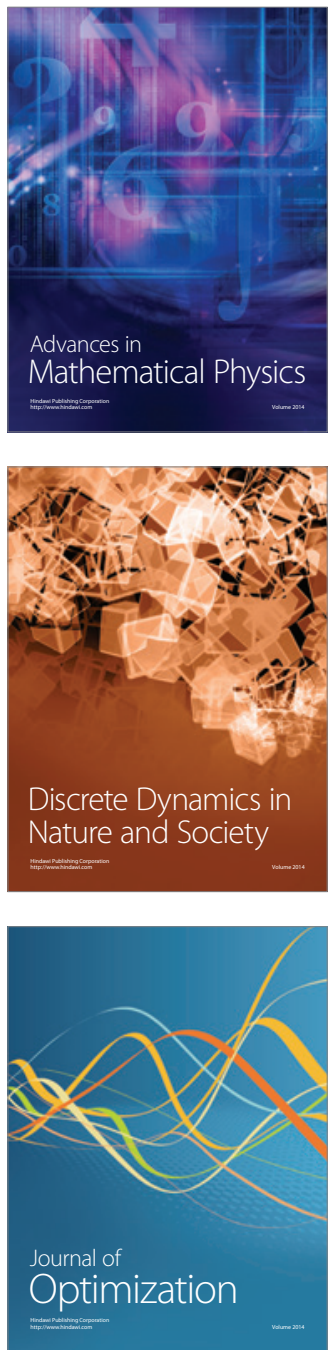